\title{
High normal blood pressure increase risks of developing adverse pregnancy outcomes
}

\author{
Dian $\mathrm{He}^{1,6, *}$, Haibing $\mathrm{Li}^{1,6, *}$, Shaowen $\mathrm{Wu}^{2, *}$, Yifei $\mathrm{Lu}^{5}$, Jun $\mathrm{Li}^{3}$, Yan He ${ }^{1,6}$, Chengchao \\ Zhang ${ }^{4}$, Zihe Zheng ${ }^{5}$ and Weiyuan Zhang ${ }^{2}$ \\ ${ }^{1}$ Department of Epidemiology and Health Statistics, School of Public Health, Capital Medical University, Beijing, China \\ ${ }^{2}$ Beijing Obstetrics and Gynecology Hospital, Capital Medical University, Beijing, China \\ ${ }^{3}$ Division of Health System, Policy and Management, JC School of Public Health and Primary Care, Chinese University of Hong \\ Kong, Sha Tin, Hong Kong \\ ${ }^{4} 302$ Military Hospital of China, Beijing Shi, China \\ ${ }^{5}$ Epidemiology Department, Johns Hopkins University Bloomberg School of Public Health, Baltimore, Maryland, USA \\ ${ }^{6}$ Beijing Municipal Key Laboratory of Clinical Epidemiology, Beijing, China \\ *These authors contributed equally to this work \\ Correspondence to: Dian He, email: hedian@ccmu.edu.cn \\ Zihe Zheng, email: zzheng11@jhu.edu \\ Weiyuan Zhang, email: zhangwy9921@hotmail.com
}

Keywords: high normal blood pressure; adverse pregnancy outcomes; gestational hypertension; preeclampsia

Received: August 14, $2017 \quad$ Accepted: November 17, $2017 \quad$ Published: January 02, 2018

Copyright: He et al. This is an open-access article distributed under the terms of the Creative Commons Attribution License 3.0 (CC BY 3.0), which permits unrestricted use, distribution, and reproduction in any medium, provided the original author and source are credited.

\section{ABSTRACT}

This study evaluated the effects of high normal blood pressure (HNBP) in early pregnancy on adverse pregnancy outcomes. We conducted a multi-center and national representative retrospective cohort study. We defined high normal blood pressure as systolic blood pressure between $130-140 \mathrm{mmHg}$ or diastolic blood pressure between $85-90 \mathrm{mmHg}$. We used multivariable logistic regression to examine the association of HNBP and risks of pregnancy outcomes. Of 69687 normotensive women in early pregnancy, 5798 (8.3\%) fulfilled our definition of HNBP, 20394 (29.3\%) were in normal blood pressure group, and the rest 43495 (62.4\%) women had optimal blood pressure. The incidence rates of gestational hypertension, preeclampsia, gestational diabetes mellitus (GDM), premature birth, small for gestational age (SGA), caesarean section, placental abruption and perinatal mortality were $1.6 \%, 2.3 \%, 4.2 \%, 6.1 \%$, $\mathbf{7 . 1} \%, \mathbf{5 4 . 9} \%, \mathbf{0 . 5} \%$ and $\mathbf{0 . 7 \%}$ respectively. Compared to women who had optimal blood pressure, those with HNBP had significantly higher odds of preeclampsia (OR = 4.179, 95\% CI 3.584, 4.873), gestational hypertension (OR $=6.050,95 \%$ CI 5.071, $7.219)$, GDM (OR $=1.077,95 \%$ CI 1.007, 1.153), premature birth (OR $=1.504,95 \%$ CI 1.329, 1.702), SGA (OR $=1.329,95 \%$ CI 1.177, 1.500) and cesarean delivery (OR $=1.583,95 \%$ CI 1.379, 1.817). Our restricted cubic spline results supported positive dose-response relationships between continuous blood pressure and the odds of these pregnancy complications. HNBP in early pregnancy significantly increased the risk of developing preeclampsia, gestational hypertension, GDM, premature birth, SGA and cesarean delivery. Our study provided robust epidemiological evidences for monitoring HNBP in early pregnancy to reduce the risks of adverse pregnancy outcomes. 


\section{INTRODUCTION}

High blood pressure (BP) in early pregnancy increases the risk of hypertensive disorders during pregnancy, cerebral hemorrhage, hepatic failure and acute renal failure in late pregnancy [1-4]. However, some studies proposed that pregnant women with high normal blood pressure (HNBP, defined as systolic blood pressure between $130-140 \mathrm{mmHg}$ or diastolic blood pressure between $85-90 \mathrm{mmHg}$ ) could also benefit from early hypertension management [5]. Previous studies found that HNBP was significantly associated with the development of gestational hypertension (adjusted odds ratio $[\mathrm{OR}] 1.81,95 \%$ confidence interval $[\mathrm{CI}] 1.16,3.25)$ and preeclampsia (adjusted OR 6.05, 95\% CI 3.46, 12.6) [6]. Other studies reported significant associations of HNBP with small for gestational age (SGA), stillbirth and high perinatal mortality $[7,8]$. However, the effect of $\mathrm{HNBP}$ on pregnancy risks is in debate. A study reported no difference in the risks of pregnancy loss, high-level neonatal care, or overall maternal complications between two groups of targeted diastolic blood pressure of $100 \mathrm{mmHg}$ versus $85 \mathrm{mmHg}[9,10]$.

Given these conflicting findings of HNBP and the risks of adverse pregnancy outcomes, more evidences are needed for clarifying the most preferable pregnancy blood pressure management in clinical practice. Thus, our primary objective is to study the associations between HNBP and common adverse pregnancy outcomes, including gestational hypertension, preeclampsia, gestational diabetes mellitus (GDM), premature birth, SGA, cesarean delivery, placental abruption and perinatal mortality. The second objective of our study is to examine the potential modification effect of pre-pregnancy body mass index (BMI) on the associations between HNBP and the above adverse outcomes.

\section{RESULTS}

Of 112386 participants, we excluded 2969 women with chronic hypertension, 15240 women missing blood pressure measurement in early pregnancy, 19531 women missing pre-pregnancy BMI, 4959 women missing GWG. A total of 69687 women were included in the analysis. We did not find significant differences between included women and excluded women in this analysis.

Of the 69, 687 participants, 5, $798(8.3 \%)$ were categorized into the HNBP group, 20, 394 (29.3\%) into the normal blood pressure group, and 43, 495 (62.4\%) into the optimal BP group (Table 1). The incidences of HNBP in the underweight, normal weight, and overweightobese groups were $4.7 \%, 7.1 \%$ and $15.5 \%$, respectively (Figure 1). Early pregnancy blood pressure levels were significantly correlated with pre-pregnancy BMI $(p<$ 0.001 ), and women with higher pre-pregnancy BMI level also had higher blood pressure level. Other maternal characteristics were significantly different across the three blood pressure groups, except for smoking status and family history of hypertension.

Among the 69, 687 normotensive pregnant women, the incidences of gestational hypertension, preeclampsia, GDM, premature birth, SGA, cesarean delivery, placental abruption and perinatal mortality were $1.6 \%, 2.3 \%, 4.2 \%$, $6.1 \%, 7.1 \%, 54.9 \%, 0.5 \%$ and $0.7 \%$ respectively. The incidence rates of these outcomes in HNBP group were higher than those in the other two groups (all $p$ values $<0.001$, in Table 2), except for placental abruption or perinatal mortality. Normotensive women with HNBP were more likely to develop preeclampsia or gestational hypertension earlier than other two groups (Figure 2).

We performed multivariable analyses to examine whether BP levels were associated with developing the above outcomes. We adjusted for maternal age, years of education, smoking, alcohol consumption, height, pre-pregnancy BMI, GWG, nulliparous status, family history of hypertension, levels of hospitals and human development index (Table 3). Results shown that pregnant women in HNBP group or the normal blood pressure group were more likely to develop preeclampsia, with odds ratios equaled to 4.179 (95\% CI $3.584,4.873)$ and $2.503(95 \%$ CI $2.215,2.828)$ respectively (Figure 3). Similarly, HNBP was significantly associated with the increased odds of gestational hypertension $(\mathrm{OR}=$ $6.050,95 \%$ CI $5.071,7.219)$, GDM (OR $=1.583,95 \%$ CI $1.379,1.817)$, premature birth $(\mathrm{OR}=1.504,95 \% \mathrm{CI} 1.329$, 1.702), $\mathrm{SGA}(\mathrm{OR}=1.329,95 \% \mathrm{CI} 1.177,1.500)$ and $\mathrm{CS}$ $(\mathrm{OR}=1.077,95 \% \mathrm{CI} 1.007,1.153)$ respectively. However, we did not observe significant associations existed between BP levels and placental abruption $(\mathrm{OR}=0.907,95 \% \mathrm{CI}$ $0.554,1.485)$ or perinatal mortality $(\mathrm{OR}=1.506,95 \% \mathrm{CI}$ $0.980,2.316)$. We also found that pre-pregnancy BMI levels were significantly associated with the risks of preeclampsia, gestational hypertension, GDM, premature birth and SGA (Table 3). However, the interaction effects of BP and prepregnancy $\mathrm{BMI}$ on above pregnancy outcomes were not statistically significant in our study (all $p$ value $>0.05$ ).

Using blood pressure as a continuous variable, multivariable logistic regression models reveal that both higher SBP and DBP were significantly associated with the increased odds of preeclampsia, the effect of which was independent of pre-pregnancy BMI (Table 4). Per 5 $\mathrm{mmHg}$ increase in SBP, there was $29 \%$ increased odds of preeclampsia $(\mathrm{OR}=1.290,95 \% \mathrm{CI} 1.254,1.327)$; and per $5 \mathrm{mmHg}$ increase in DBP, there was $46.6 \%$ increased odds of preeclampsia (OR $=1.466,95 \%$ CI 1.409, 1.525). Associations were observed between SBP/ DBP with gestational hypertension, GDM, premature birth, SGA and cesarean delivery (Table 4). In order to test the robustness of our findings, we restricted our analysis to women with BMI between $18.5 \mathrm{~kg} / \mathrm{m}^{2}$ and $24.9 \mathrm{~kg} / \mathrm{m}^{2}(n=44673)$, and results in this subgroup were consistent with the overall population (Table 4).

Using SBP equals to $120 \mathrm{mmHg}$ or DBP equals to $80 \mathrm{mmHg}$ groups as the reference, we applied restricted 
cubic spline regression to analyze the dose-response relationship between continuous blood pressure change (SBP and DBP) and the odds of the above pregnancy outcomes (Figure 4). Based on the blood pressure-outcome association trajectory, we found that there were significant nonlinear dose-response relationships between the adverse pregnancy outcomes and the continuous blood pressure change. Compared to other outcomes, preeclampsia and gestational hypertension had stronger dose-response relationship (steeper trajectory) with blood pressure level (Figure 4).

\section{DISCUSSION}

In this large, national representative retrospective cohort study, we found that blood pressure level of normotensive women was associated with the development of gestational hypertension, preeclampsia, GDM, premature birth, SGA and caesarean section, where stronger associations were seen with gestational hypertension and preeclampsia. Higher blood pressure increased women's risks of developing adverse pregnancy outcomes [11]. Results of sensitivity analyses were consistent with our main findings. Although pre-pregnant BMI was also an independent risk factor for above pregnancy outcomes, we did not observe significant interaction effects of blood pressure with pre-pregnancy BMI on the outcomes.

We found stronger associations between BP level in early pregnancy and the development of preeclampsia and gestational hypertension. After adjusting for confounders, the risks of preeclampsia or gestational hypertension were 2.5-6 times higher comparing women in the normal blood pressure or HNBP group to those with optimal blood pressure. A retrospective cohort study demonstrated that the adjusted ORs of normal blood pressure and HNBP for the subsequent occurrence of preeclampsia were 5.1 (95\% CI $2.2,12)$ and $8.3(95 \%$ CI $3.1,22)$ respectively; and the adjusted ORs for gestational hypertension were 7.0 $(95 \%$ CI $2.6,19)$ and $7.4(95 \%$ CI $2.1,25)$ respectively [12]. Several studies showed that blood pressure level of early pregnancy in women who developed preeclampsia or gestational hypertension at a later time was higher than those who did not $[12,13]$. Magee's study reported that women in the group with less-tight blood pressure control group (targeted diastolic blood pressure of $100 \mathrm{mmHg}$ ) had a significantly higher frequency of severe maternal hypertension than those in the tighter control group (85 $\mathrm{mmHg}$ ) [9]. Additional evidence showed that tighter blood pressure control during pregnancy brings benefit to mothers without increasing the neonates' risks, along with lowering the cost of pregnancy health care [5]. Thence, some researcher proposed that a cut off value of DBP equaled to $81 \mathrm{mmHg}$ was the optimal threshold for early gestational blood pressure level, which allowed for identifying pregnant women with higher risks of gestational [14].

In addition, we also found that women with higher blood pressure level were more likely to develop preeclampsia or gestational hypertension at earlier time than those with lower blood pressure level. Previous studies showed that chronic hypertension was a wellestablished risk factor for developing preeclampsia prior to 34 th gestational week $[15,16,17]$. In our study, women in the HNBP group had higher percentage developing preeclampsia or gestational hypertension before 32 weeks of gestation (Figure 2).

We observed that the incidence of SGA neonates was 1.3 times higher in the HNBP group compared to the optimal blood pressure group. A previous study suggested the risk of SGA had 1.8-fold increase among mothers with high blood pressure even when preeclampsia was absent

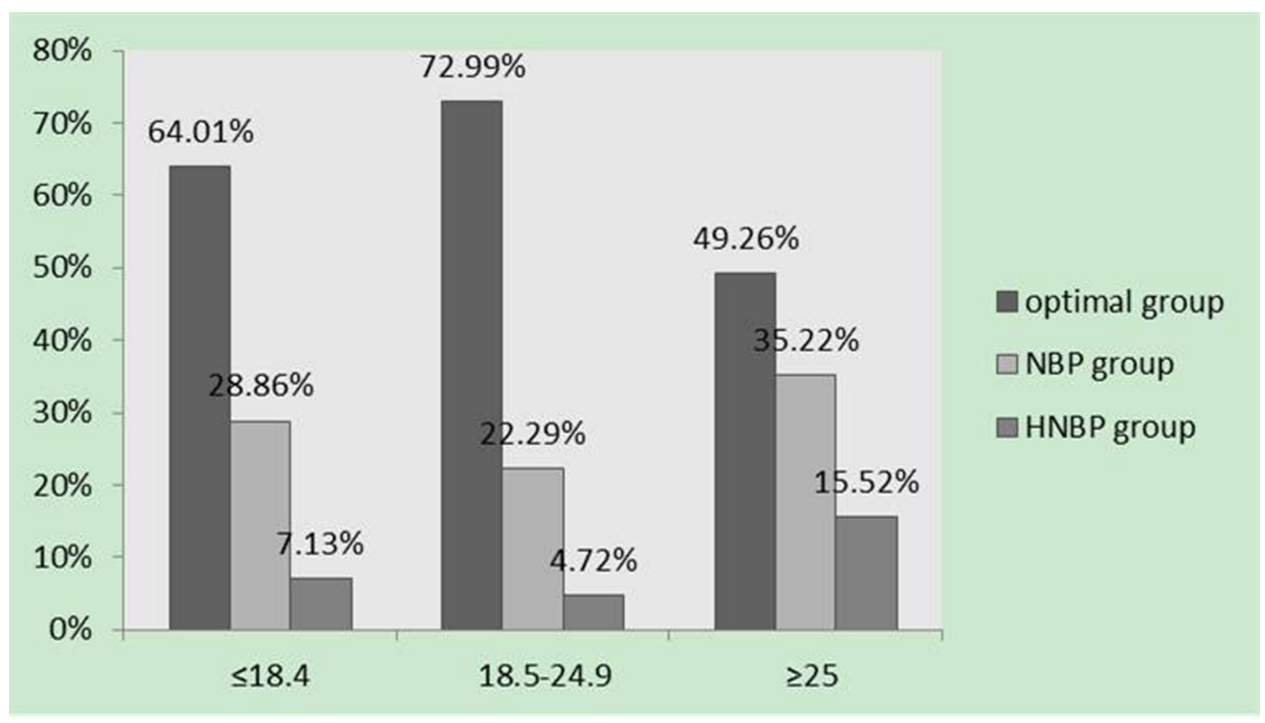

Figure 1: Proportions of blood pressure levels in body mass index groups (NBP: normal blood pressure; HNBP: high normal blood pressure). 
[11]. Anna-Karin et al. found that prehypertension was associated with increased risk of SGA birth with adjusted OR of 1.69 (95\% CI 1.51, 1.90). Additionally, risks of SGA birth increased by $2.0 \%(95 \%$ CI $1.5,2.8)$ per each one $\mathrm{mm} \mathrm{Hg}$ rise in DBP during pregnancy [8]. The possible biological rationale for this association is that high blood pressure, along with placental gene expression and function, affects critical components of maternal metabolism [18]. Study found that impaired maternal perfusion of the placenta (an extrinsic defect) and impaired placental development (an intrinsic defect) could cause SGA [19].

We also observed associations between HNBP and the development of preterm birth and cesarean section. To our best knowledge, there was no study reported the association between HNBP with preterm birth or cesarean section. However, studies indicated that, even in the absence of superimposed preeclampsia, women with chronic hypertension had an increased risk of preterm or cesarean delivery [20]. Panaitescu's study indicated that chronic hypertension was associated with 3.7-fold increased risk of iatrogenic preterm birth and 1.8-fold increased risk of cesarean section [11]. A meta-analysis studies showed that women with chronic hypertension had 30\% higher risks of cesarean delivery $(\mathrm{OR}=1.3,95 \% \mathrm{CI} 1.1,1.5)$, and $170 \%$ increased risks for preterm delivery $(\mathrm{OR}=2.7,95 \% \mathrm{CI} 1.9$, 3.6) [21]. However, the pathogenesis of spontaneous preterm birth among women with hypertension remains unclear.

Among our normotensive study population, we found positive association between blood pressure and the risks of GDM. There was no study explored the association between HNBP and the development of GDM among normotensive pregnancy women. A prospective study based on 109,932 pregnancies shown that chronic hypertension was associated with increased risk of GDM $(\mathrm{OR}=1.61,95 \%$ CI 1.27, 2.05) [11]. Another study reported that gestational hypertension was associated with increased risk of post-delivery diabetes mellitus $(\mathrm{HR}=$ $1.52,95 \%$ CI 1.21, 1.89) [3]. Insulin resistance, chronic inflammation and endothelial dysfunction may contribute to the biological rationale of the association [21-22].

The associations between blood pressure levels and occurrences of placental abruption (OR $=0.907,95 \% \mathrm{CI}$ $0.554,1.485)$ or perinatal mortality $(\mathrm{OR}=1.506,95 \% \mathrm{CI}$ $0.980,2.316$ ) were not statistically significant in our study. A previous study found that each $\mathrm{mmHg}$ increase in DBP from early to late pregnancy did not alter the risk of stillbirth (95\% CI-1.4, 1.7) [8]. As far as we known, few publications had focuses on the association of HNBP and placental abruption. Researchers proposed that high blood pressure might lead to an imbalance in proangiogenic and antiangiogenic factors, notably an increase in soluble fms-like tyrosine kinsase-1 (sFlt-1), thereby leading to placental abruption [23].

The prevalence of high blood pressure among pregnant women has been increasing over time. This is primarily attributed to the increased prevalence of obesity and the delayed childbearing age [24]. A large number of pregnancy women unaware of their blood pressure level or HNBP condition. Early health education and counseling about the risks of hypertensive disease during pregnancy are needed for prospective mothers. Our findings provided robust epidemiological evidences and new insight to the relationship between maternal blood pressure in early pregnancy and adverse pregnancy outcomes. Based on this large and national representative cohort study, we recommended that health care providers should spend more efforts on the monitoring and follow-up with HNBP women during early pregnancy, especially for those who are overweight or older.

Our study had several strengths. First, we obtained the information of blood pressure, weight, height,

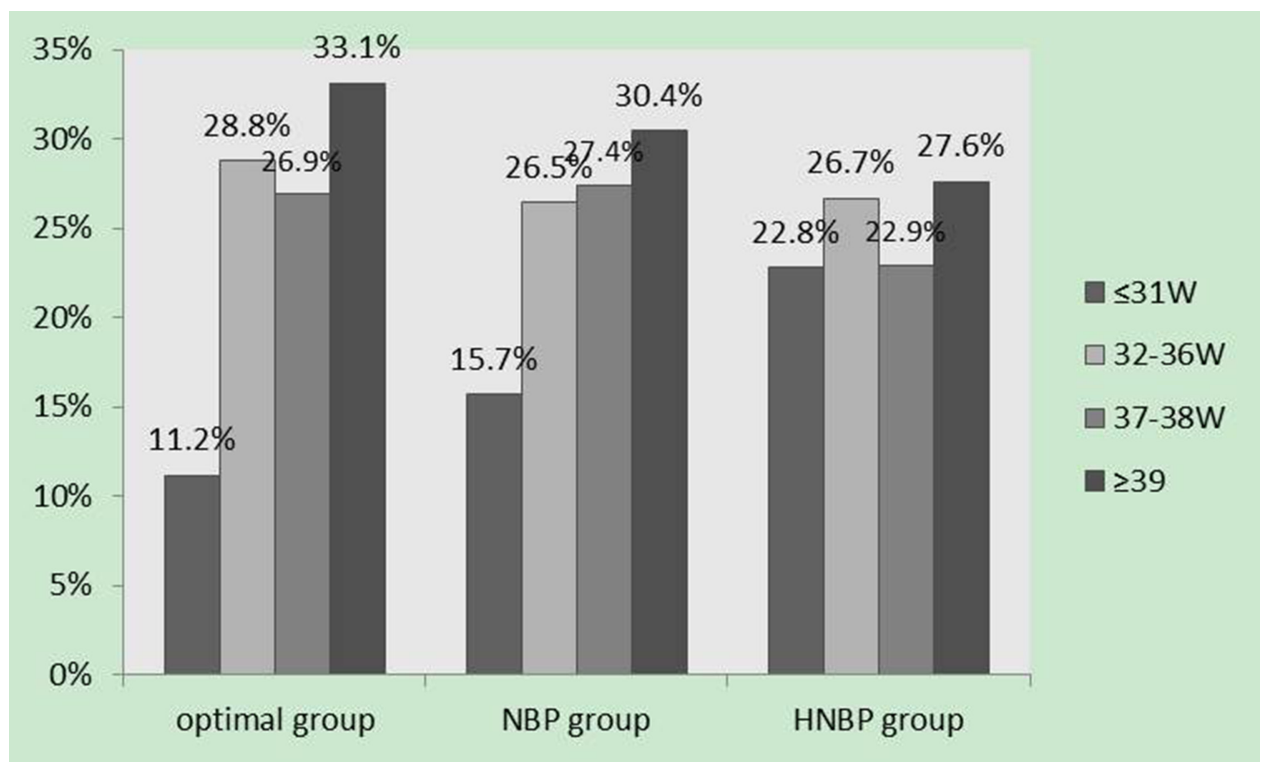

Figure 2: Proportions of onset time classifications of PE or GH in blood pressure groups (W= weeks; NBP: normal blood pressure; HNBP: high normal blood pressure). 
gestational age, and adverse pregnancy outcomes directly from clinic medical records from each research center, which was an objective data source compared to selfreport. Second, we are confident that the classification of blood pressure categories was accurate since the measurements were conducted at the time of women's first hospital visit. Third, the national representative nature of our study population lowered the cohort selection bias and increased the external validity of our conclusion.

The limitations of our study are as following. In this study, we did not to examine the effect of HNBP on the onset time of adverse outcomes that occurred during pregnancy. In future studies, we would like to prospectively evaluate and quantify the relationship between pre-pregnancy blood pressure level and the onset time of different adverse outcomes, especially preeclampsia and gestational hypertension. Second, we were not able to conclude causality, but associations, between blood pressure and pregnancy complications. Third, since the study was based on Chinese population, readers are advised to be careful when generalizing the results to other circumstance.

In conclusion, these results provide robust epidemiological evidences: high normal blood pressure of normotensive pregnancy women significantly increased risks of development of gestational hypertension, preeclampsia, GDM, premature birth, SGA and caesarean section, where the risks increased by 5 times for developing gestational hypertension, and increased by 3 times for preeclampsia. HNBP in combination with other clinical indicators can predict the probability of occurrence of these adverse pregnancy outcomes. Thence, we

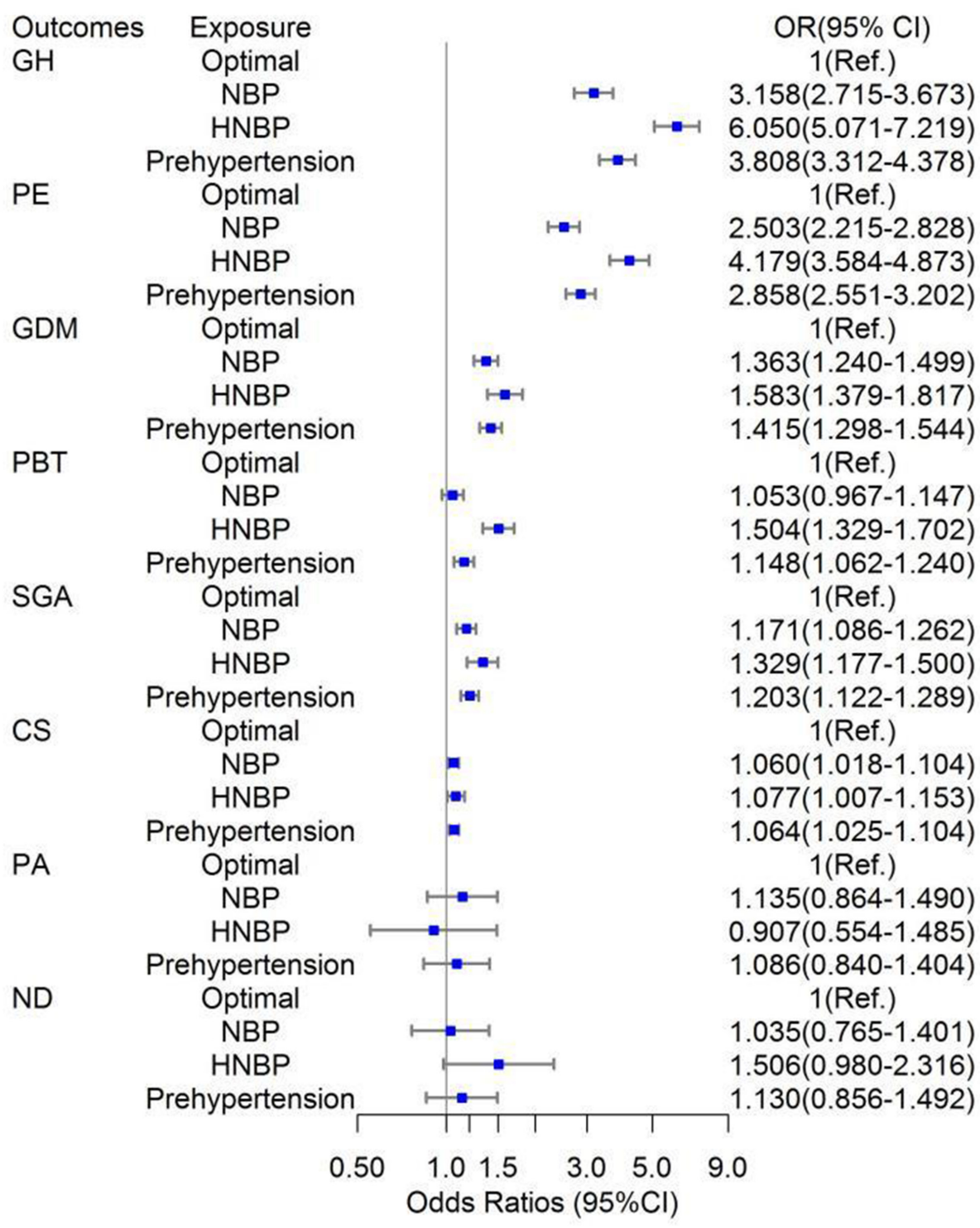

Figure 3: Odds ratios of high-normal, normal, prehypertension groups associated with occurrences of adverse pregnancy outcomes. (GH: Gestational hypertension; PE: Pre-eclampsia; GDM: Gestational Diabetes Mellitus; PB: Premature birth; SGA: small for gestational age; CS: Caesarean Section; NBP: normal blood pressure; HNBP: high normal blood pressure; prehypertension: HNBP or NBP). 
recommend health care providers increase the monitoring for high normal blood pressure during early pregnancy in the clinical practice.

\section{MATERIALS AND METHODS}

\section{Study design}

We conducted a retrospective study using a multicenter and national representative cohort study, which was originally designed for investigating the prevalence of common pregnancy and birth complications in China. In the retrospective study, data on the relevant events for each individual are collected from existing records, and can immediately be analyzed to determine the relative risk of the cohort compared to the control group. In 2011, 38 general hospitals or gynecology and obstetrics specialty hospitals were selected from 14 provinces and regions across China. These research sites covered most provinces in China and provided more than
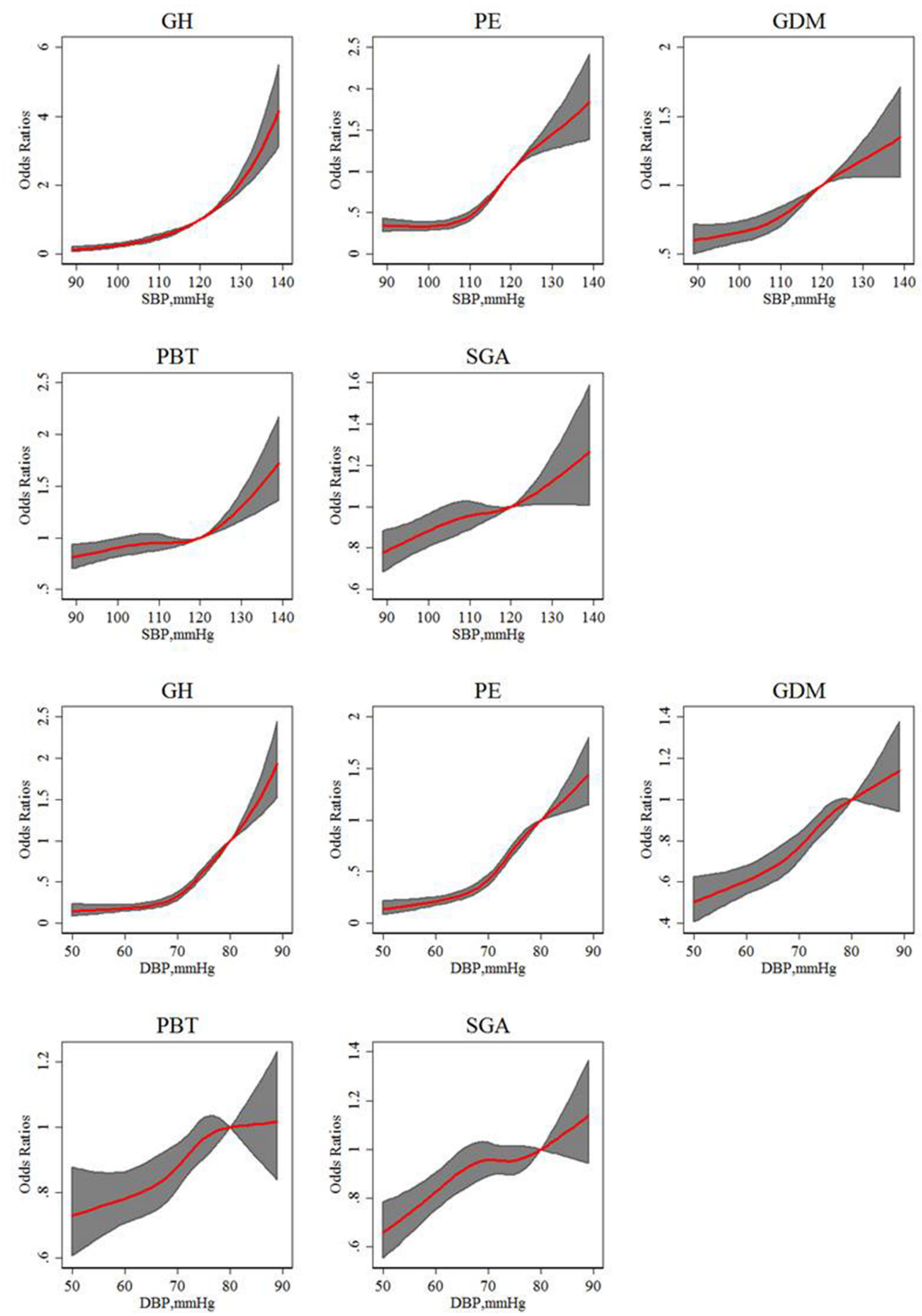

Figure 4: Risk of pregnancy outcomes according to SBP or DBP as a continuous variable. Gray areas are $95 \%$ confidence intervals. Odds ratios were estimated using logistic regression modeling, adjusting for maternal age, years of education, smoking, alcohol consumption, height, pre-pregnancy BMI, GWG, nulliparous, family history of hypertension, levels of hospitals and HDI. (GH: Gestational hypertension; PE: Pre-eclampsia; GDM: Gestational Diabetes Mellitus; PB: Premature birth; SGA: small for gestational age; BMI: body mass index; GWG: gestational weight gain; HDI: human development index). 
Table 1: Maternal characteristics in three groups divided by the blood pressure value around 12 weeks of pregnancy $(N=69687)$

\begin{tabular}{|c|c|c|c|c|}
\hline & Optimal & Normal & High normal & $p$-value \\
\hline & $43495(62.4 \%)$ & $20394(29.3 \%)$ & $5798(8.3 \%)$ & \\
\hline Age (years) & & & & $<0.001$ \\
\hline$\leq 24$ & $9877(22.8)$ & 4999 (24.6) & $1223(21.3)$ & \\
\hline $25-29$ & $18934(43.8)$ & $8448(41.7)$ & $2320(40.3)$ & \\
\hline $30-34$ & $10698(24.7)$ & $4753(23.4)$ & $1525(26.5)$ & \\
\hline$\geq 35$ & $3720(8.6)$ & $2080(10.3)$ & $686(11.9)$ & \\
\hline Years of education & & & & $<0.001$ \\
\hline$\geq 13$ & $21273(49.6)$ & $8081(40.3)$ & $2428(42.6)$ & \\
\hline $10-12$ & $12846(29.9)$ & $6812(34.0)$ & $1912(33.5)$ & \\
\hline$\leq 9$ & $8798(20.5)$ & $5139(25.7)$ & $1361(23.9)$ & \\
\hline Smoking & & & & 0.984 \\
\hline Smoker & $153(0.4)$ & $70(0.3)$ & $20(0.3)$ & \\
\hline Non-smoker & $43342(99.6)$ & $20324(99.7)$ & $5778(99.7)$ & \\
\hline Drinking & & & & $<0.001$ \\
\hline No & $42835(98.5)$ & $20175(98.9)$ & $5743(99.1)$ & \\
\hline Yes & $660(1.5)$ & $219(1.1)$ & $55(0.9)$ & \\
\hline Height (cm) & & & & $<0.001$ \\
\hline$\leq 159$ & $10924(28.2)$ & $4693(26.5)$ & $1208(24.6)$ & \\
\hline $160-164$ & $17812(45.9)$ & $7958(44.9)$ & $2232(45.4)$ & \\
\hline $165-169$ & $8130(21.0)$ & $4067(22.9)$ & $1138(23.2)$ & \\
\hline$\geq 170$ & 1908 (4.9) & $1023(5.8)$ & $335(6.8)$ & \\
\hline Pre-pregnancy BMI & & & & $<0.001$ \\
\hline$\leq 18.4$ & $5227(14.1)$ & $1596(9.5)$ & $338(7.4)$ & \\
\hline $18.5-24.9$ & $28593(76.9)$ & $12893(76.3)$ & $3187(69.6)$ & \\
\hline$\geq 25$ & $3355(9.0)$ & $2399(14.2)$ & $1057(23.1)$ & \\
\hline GWG & & & & $<0.001$ \\
\hline Below & $9114(25.4)$ & 4558 (27.6) & $1098(24.9)$ & \\
\hline Adequate & $15360(42.9)$ & $6605(40.0)$ & $1737(39.5)$ & \\
\hline Above & $11367(31.7)$ & $5348(32.4)$ & $1567(35.6)$ & \\
\hline Nulliparous & & & & $<0.001$ \\
\hline Yes & $36563(84.1)$ & $16682(81.8)$ & $4809(82.9)$ & \\
\hline No & $6932(15.9)$ & $3712(18.2)$ & $989(17.1)$ & \\
\hline Family history of hypertension & & & & 0.070 \\
\hline No & $42804(98.4)$ & $20115(98.6)$ & $5701(98.3)$ & \\
\hline Yes & $691(1.6)$ & $279(1.4)$ & $97(1.7)$ & \\
\hline Levels of hospitals & & & & $<0.001$ \\
\hline Two-level & $12504(28.7)$ & $7060(34.6)$ & $1751(30.2)$ & \\
\hline Three-level & $30991(71.3)$ & $13334(65.4)$ & $4047(69.8)$ & \\
\hline HDI & & & & $<0.001$ \\
\hline High-level & $24558(56.5)$ & $7907(38.8)$ & $2550(44.0)$ & \\
\hline Middle-level & $6418(14.8)$ & $4842(23.7)$ & $1111(19.2)$ & \\
\hline Low-level & $12519(28.8)$ & $7645(37.5)$ & $2137(36.9)$ & \\
\hline
\end{tabular}

BMI: body mass index $\left(\mathrm{kg} / \mathrm{m}^{2}\right)$; GWG: gestational weight gain; HDI: Human Development Index. 
Table 2: Percentage of maternal and neonatal outcomes stratified by the blood pressure value around 12 weeks of pregnancy $(N=69687)$

\begin{tabular}{lllll}
\hline & Optimal & Normal & High normal & $p$-value \\
\cline { 2 - 5 } & $\mathbf{N = 4 3 4 9 5 ( \% )}$ & $\boldsymbol{N}=\mathbf{2 0 3 9 4}(\mathbf{\%})$ & $\boldsymbol{N}=\mathbf{5 7 9 8}(\mathbf{\%})$ & \\
\hline Gestational hypertension & $358(0.8)$ & $460(2.3)$ & $284(4.9)$ & $<0.001$ \\
Pre-eclampsia & $581(1.3)$ & $705(3.5)$ & $337(5.8)$ & $<0.001$ \\
Gestational diabetes & $1636(3.8)$ & $919(4.5)$ & $364(6.3)$ & $<0.001$ \\
Premature Birth & $2500(5.8)$ & $1294(6.3)$ & $451(7.8)$ & $<0.001$ \\
SGA & $2828(6.7)$ & $1552(7.8)$ & $472(8.3)$ & $<0.001$ \\
Caesarean Section & $23025(52.9)$ & $11800(57.9)$ & $3455(59.6)$ & $<0.001$ \\
Placental Abruption & $196(0.5)$ & $105(0.5)$ & $28(0.5)$ & 0.540 \\
Perinatal mortality & $303(0.7)$ & $164(0.8)$ & $47(0.8)$ & 0.263 \\
\hline
\end{tabular}

Pre-eclampsia: including mild and severe preeclampsia; SGA: small for gestational age; Perinatal mortality: including neonatal death and stillbirth.

Table 3: Odds ratios of blood pressure levels or Pre-pregnancy BMI levels associated with occurrences of adverse pregnancy outcomes

\begin{tabular}{lccccccc}
\hline & \multicolumn{5}{c}{ Blood pressures } & Pre-pregnancy BMI & Anteraction \\
& \cline { 2 - 6 } & \multicolumn{3}{c}{ AOR (95\%CI) } & \multicolumn{2}{c}{ AOR (95\%CI) } & P value \\
\cline { 2 - 6 } & Optimal & Normal & High-normal & Normal & Below & Above & \\
\hline Gestational & & 3.158 & 6.050 & & 0.786 & 1.605 & 0.454 \\
hypertension & 1 (Ref.) & $(2.715-3.673)$ & $(5.071-7.219)$ & 1 (Ref.) & $(0.612-1.009)$ & $(1.372-1.877)$ & \\
Pre- & & 2.503 & 4.179 & & 0.937 & 1.391 & 0.157 \\
eclampsia & 1 (Ref.) & $(2.215-2.828)$ & $(3.584-4.873)$ & 1 (Ref.) & $(0.770-1.140)$ & $(1.209-1.600)$ & \\
Gestational & & 1.363 & 1.583 & & 0.683 & 2.113 & 0.081 \\
Diabetes & 1 (Ref.) & $(1.240-1.499)$ & $(1.379-1.817)$ & 1 (Ref.) & $(0.584-0.798)$ & $(1.900-2.350)$ & \\
Premature & & 1.053 & 1.504 & & 1.259 & 1.051 & 0.051 \\
Birth & 1 (Ref.) & $(0.967-1.147)$ & $(1.329-1.702)$ & 1 (Ref.) & $(1.128-1.405)$ & $(0.934-1.183)$ & \\
& & 1.171 & 1.329 & & 1.610 & 0.712 & 0.058 \\
SGA & 1 (Ref.) & $(1.086-1.262)$ & $(1.177-1.500)$ & 1 (Ref.) & $(1.472-1.760)$ & $(0.624-0.813)$ & \\
Caesarean & & 1.060 & 1.077 & & 0.752 & 1.593 & 0.521 \\
Section & 1 (Ref.) & $(1.018-1.104)$ & $(1.007-1.153)$ & 1 (Ref.) & $(0.713-0.793)$ & $(1.499-1.692)$ & \\
Placental & & 1.135 & 0.907 & & 1.087 & 0.726 & 0.859 \\
Abruption & 1 (Ref.) & $(0.864-1.490)$ & $(0.554-1.485)$ & 1 (Ref.) & $(0.754-1.567)$ & $(0.474-1.114)$ & \\
Perinatal & & 1.035 & 1.506 & & 1.141 & 0.997 & 0.259 \\
mortality & 1 (Ref.) & $(0.765-1.401)$ & $(0.980-2.316)$ & 1 (Ref.) & $(0.744-1.750)$ & $(0.627-1.585)$ & \\
\hline
\end{tabular}

Pre-eclampsia: including mild and severe preeclampsia; SGA: small for gestational age; Perinatal mortality: including neonatal death and stillbirth; ${ }^{*}$ Interaction $p$ value: the interaction effect between blood pressure and pre-pregnancy BMI associated with adverse pregnancy outcomes; AOR: adjusted odds ratio.

120000 women's records. The chosen hospitals included 19 tertiary hospitals and 19 secondary hospitals, which are the most common medical institutions in mainland China.

\section{Data collection}

We included pregnant women who delivered babies during January 1st 2011 and December 31st 2011 in the selected hospitals as study population. We collected participants' clinical information from their clinical medical records. Data collection was conducted by trained medical staffs and all the collected information was crosschecked by study investigators for quality control. These clinical medical records include sociodemographic information, disease history, the occurrence of pregnancy complications, as well as perinatal and neonatal outcomes. Data had been routinely updated from participants' first prenatal visit (around 12th gestational week). The detailed 
Table 4: Odds ratios of SBP or DBP by increment of $5 \mathrm{mmHg}$ associated with occurrences of adverse pregnancy outcomes by categories of pre-pregnancy BMI

\begin{tabular}{|c|c|c|c|c|}
\hline & \multicolumn{2}{|c|}{ Overall population } & \multicolumn{2}{|c|}{ Population with $18.5 \leq$ BMI $<24.9$} \\
\hline & $\begin{array}{c}\text { SBP } \\
\text { OR }(95 \% \mathrm{CI})\end{array}$ & $\begin{array}{c}\text { DBP } \\
\text { OR }(95 \% \mathrm{CI})\end{array}$ & $\begin{array}{c}\text { SBP } \\
\text { OR }(95 \% \mathrm{CI})\end{array}$ & $\begin{array}{c}\text { DBP } \\
\text { OR }(95 \% C I)\end{array}$ \\
\hline Gestational hypertension & $\begin{array}{c}1.444 \\
(1.396-1.494)\end{array}$ & $\begin{array}{c}1.592 \\
(1.520-1.668)\end{array}$ & $\begin{array}{c}1.459 \\
(1.400-1.521)\end{array}$ & $\begin{array}{c}1.604 \\
(1.516-1.696)\end{array}$ \\
\hline Pre-eclampsia & $\begin{array}{c}1.290 \\
(1.254-1.327)\end{array}$ & $\begin{array}{c}1.466 \\
(1.409-1.525)\end{array}$ & $\begin{array}{c}1.311 \\
(1.268-1.356)\end{array}$ & $\begin{array}{c}1.476 \\
(1.409-1.547)\end{array}$ \\
\hline Gestational Diabetes & $\begin{array}{c}1.102 \\
(1.080-1.124)\end{array}$ & $\begin{array}{c}1.140 \\
(1.110-1.170)\end{array}$ & $\begin{array}{c}1.090 \\
(1.065-1.117)\end{array}$ & $\begin{array}{c}1.122 \\
(1.088-1.158)\end{array}$ \\
\hline Premature Birth & $\begin{array}{c}1.050 \\
(1.032-1.069)\end{array}$ & $\begin{array}{c}1.055 \\
(1.029-1.080)\end{array}$ & $\begin{array}{c}1.047 \\
(1.025-1.069)\end{array}$ & $\begin{array}{c}1.069 \\
(1.039-1.099)\end{array}$ \\
\hline SGA & $\begin{array}{c}1.044 \\
(1.028-1.061)\end{array}$ & $\begin{array}{c}1.059 \\
(1.037-1.082)\end{array}$ & $\begin{array}{c}1.042 \\
(1.023-1.061)\end{array}$ & $\begin{array}{c}1.064 \\
(1.037-1.091)\end{array}$ \\
\hline Caesarean Section & $\begin{array}{c}1.002 \\
(0.994-1.011)\end{array}$ & $\begin{array}{c}1.026 \\
(1.014-1.037)\end{array}$ & $\begin{array}{c}1.005 \\
(0.995-1.014)\end{array}$ & $\begin{array}{c}1.029 \\
(1.016-1.042)\end{array}$ \\
\hline Placental Abruption & $\begin{array}{c}1.00 \\
(0.945-1.058)\end{array}$ & $\begin{array}{c}1.040 \\
(0.962-1.125)\end{array}$ & $\begin{array}{c}1.025 \\
(0.960-1.095)\end{array}$ & $\begin{array}{c}1.050 \\
(0.960-1.148)\end{array}$ \\
\hline Perinatal mortality & $\begin{array}{c}1.071 \\
(1.000-1.147) \\
\end{array}$ & $\begin{array}{c}1.073 \\
(0.977-1.180) \\
\end{array}$ & $\begin{array}{c}1.051 \\
(0.972-1.136) \\
\end{array}$ & $\begin{array}{c}1.075 \\
(0.966-1.196) \\
\end{array}$ \\
\hline
\end{tabular}

Pre-eclampsia: including mild and severe preeclampsia; SGA: small for gestational age; Perinatal mortality: including neonatal death and stillbirth; BMI: pre-pregnancy BMI; SBP: Systolic BP; DBP: Diastolic BP.

study design can be found in previous publication [25].

This study has been approved by the Ethics Committees of each selected hospital, and followed the guidelines of the Helsinki agreement and its amendments. The National Research Ethics Service had previously approved the anonymous use of these data for research purposes.

\section{Definition of exposures}

Blood pressure was measured around the 12th gestational week using electronic sphygmomanometer or mercury sphygmomanometer with patients in the sitting position holding her right arm at heart level. Applying conservative exposure definition, we used the lower blood pressure reading for individuals who had more than one blood pressure measurement [26]. We categorized participants into 3 groups according to their blood pressure level as optimal (SBP $<120 \mathrm{mmHg}$ and $\mathrm{DBP}<80 \mathrm{mmHg}$ ), normal $(130 \mathrm{mmHg}>\mathrm{SBP} \geq 120 \mathrm{mmHg}$ or $85 \mathrm{mmHg}$ $>$ DBP $\geq 80 \mathrm{mmHg}$ ), and high-normal $(140 \mathrm{mmHg}>\mathrm{SBP}$ $\geq 130 \mathrm{mmHg}$ or $90 \mathrm{mmHg}>\mathrm{DBP} \geq 85 \mathrm{mmHg}$ ). Normal or high-normal group was further defined as prehypertension group. Women with high blood pressure (SBP $\geq 140 \mathrm{mmHg}$ or $\mathrm{DBP} \geq 90 \mathrm{mmHg}$ ) were excluded from our analysis [4].

\section{Definition of outcomes}

The outcomes of interest in our study were gestational hypertension, preeclampsia, GDM, premature birth, SGA, cesarean delivery, placental abruption and perinatal mortality. According to the classification system by the National High Blood Pressure Education Program (NHBPEP) Working Group, hypertension disorder during pregnancy was categorized into six subtypes: gestational hypertension $(\mathrm{GH})$, mild preeclampsia, severe preeclampsia, eclampsia, preeclampsia superimposed on chronic hypertension and chronic hypertension in pregnancy [27]. Gestational hypertension was defined as hypertension without proteinuria occurring after the 20th week of gestation (ICD-9 codes 642D and 642X, and ICD-10 code O13). We combined mild preeclampsia and severe preeclampsia cases as preeclampsia (ICD-9 codes 642E-642H and ICD-10 codes O11 and O14)2. GDM was diagnosed using the oral glucose tolerance test at 24-28 weeks of pregnancy in accordance with the IADPSG criteria (ICD-9 code 648W and ICD-10 code O244) [28]. SGA was determined as infants who had birth weights below the 10th percentile of Chinese birth weight reference curve, adjusted for gestational age and gender. Premature birth was defined as childbirth occurred prior to 37 completed gestational weeks. We included stillbirth and neonatal death in the analysis of perinatal mortality.

\section{Potential confounders}

Pre-pregnancy BMI $\left(\mathrm{kg} / \mathrm{m}^{2}\right)$ was calculated using maternal weight and height recorded around $12^{\text {th }}$ gestational week. We categorized BMI into underweight $\left(<18.5 \mathrm{~kg} / \mathrm{m}^{2}\right)$, normal weight (18.5-24.9 kg/m²), overweight and obese $(\geq$ $25.0 \mathrm{~kg} / \mathrm{m}^{2}$ ) [29]. Because less than $2 \%$ of our study population 
was obese, we combined the overweight and obese groups to increase statistical power. We calculated gestational weight gain (GWG) as the difference between maternal weight prior to delivery and maternal weight recorded at the first prenatal visit (around 12th gestational week). We categorized GWG into adequate, inadequate and excessive weight gain groups based on the 2009 IOM GWG guidelines [30].

Other potential confounders that we included and adjusted in our analysis were maternal age at children birth, maternal years of education, smoking status, drinking status, height, whether nulliparous, family history of hypertension, type of hospital (tertiary versus secondary hospitals) and human development index (HDI).

\section{Statistical analysis}

We examined the difference of maternal characteristics across different blood pressure groups using chi ${ }^{2}$ test. We used multivariable logistic regression model to explore the associations between blood pressure in early pregnancy and the odds of pregnancy outcomes. Multivariable logistic regression analysis was performed to determine independent risk factors for the development of pregnancy outcomes. We also examined the interaction effect of blood pressure and pre-pregnancy BMI categories on the risks of pregnancy outcomes. Then we used SBP and DBP as continuous variables to analyze the association between continuous BP (per $5 \mathrm{mmHg}$ increase) and pregnancy outcomes. Additionally, we conducted sensitivity analyses restricted to women whose BMI ranged from $18.5 \mathrm{~kg} / \mathrm{m}^{2}$ to $24.9 \mathrm{~kg} / \mathrm{m}^{2}$ in order to validate the associations between BP with pregnancy outcomes. We used restricted cubic spline regression to analyze the dose-response relationship between continuous blood pressure and the odds ratios of individual pregnancy outcomes. In multivariable regression models, we selected potential confounding factors based on clinicians' opinions and positive results in univariate analyses $(P<0.05)$. Twosided $P<0.05$ was used as the cut-off value for statistical significance. We used SAS software (version 9.4, SAS Institute Inc, Cary, NC) for all analyses.

\section{Abbreviations}

GH: Gestational hypertension; PE: Pre-eclampsia; GDM: Gestational diabetes mellitus; PBT: Premature birth; SGA: Small for gestational age; CS: Caesarean section; PA: Placental abruption; NBP: Normal blood pressure; HNBP: High normal blood pressure; Prehypertension: HNBP or NBP; BMI: body mass index; GWG: Gestational weight gain; HDI: Human development index; SBP: Systolic BP; DBP: Diastolic BP; AOR: Adjusted odds ratio.

\section{Author contributions}

Shaowen $\mathrm{Wu}$, Jun $\mathrm{Li}$ and Yifei $\mathrm{Lu}$ directed data collection and management. Haibing $\mathrm{Li}$ and Dian $\mathrm{He}$ participated in data analysis and interpretation. Yan He and Weiyuan Zhang coordinated and supervised this investigation. Dian He, Chengchao Zhang and Zihe Zheng participated in drafting of this manuscript.

\section{CONFLICTS OF INTEREST}

The authors declare no conflicts of interest.

\section{FUNDING}

This research was supported by: The Natural Science Foundation of China (NSFS) [no. 81402689]; Beijing Municipal Science\& Techology (Z151100004015182).

\section{REFERENCES}

1. Duley L. The global impact of pre-eclampsia and eclampsia. Semin Perinatol. 2009; 33:130-137.

2. Villamor E, Cnattingius S. Interpregnancy weight change and risk of adverse pregnancy outcomes: a populationbased study. Lancet. 2006; 368:1164-70.

3. Männistö $T$, Mendola $P$, Vääräsmäki $M$, Järvelin $M R$, Hartikainen AL, Pouta A, Suvanto E. Elevated blood pressure in pregnancy and subsequent chronic disease risk. Circulation. 2013; 127:681-90.

4. Gabb GM, Mangoni AA, Arnolda L. Guideline for the diagnosis and management of hypertension in adults- 2016. Med J Aust. 2017; 206:141.

5. Ahmed RJ, Gafni A, Hutton EK, Hu ZJ, Pullenayegum E, von Dadelszen P, Rey E, Ross S, Asztalos E, Murphy KE, Menzies J, Sanchez JJ, Ganzevoort W, et al. The Cost Implications of Less Tight Versus Tight Control of Hypertension in Pregnancy (CHIPSTrial). Hypertension. 2016; 68:1049-55.

6. Mabuchi A, Yamamoto R, Ishii K, Kakubari R, Takaoka S, Muto H, Mitsuda N. Significance of high-normal blood pressure during early second trimester for predicting the onset of hypertensive disorders in pregnancy. Hypertens Pregnancy. 2016; 35:234-41.

7. Steer PJ, Little MP, Kold-Jensen T, Chapple J, Elliott P. Maternal blood pressure in pregnancy, birth weight, and perinatal mortality in first births: prospective study. BMJ. 2004; 329:1312.

8. Wikström AK, Gunnarsdottir J, Nelander M, Simic M, Stephansson O, Cnattingius S. Prehypertension in Pregnancy and Risks of Small for Gestational Age Infant and Stillbirth. Hypertension. 2016; 67:640-6.

9. Magee LA, von Dadelszen P, Rey E, Ross S, Asztalos E, Murphy KE, Menzies J, Sanchez J, Singer J, Gafni A, Gruslin A, Helewa M, Hutton E, et al. Less-tight versus tight control of hypertension in pregnancy. N Engl J Med. 2015; 372:407-17.

10. Magee LA, von Dadelszen P, Singer J, Lee T, Rey E, Ross S, Asztalos E, Murphy KE, Menzies J, Sanchez J, Gafni 
A, Helewa M, Hutton E, et al. The CHIPS Randomized Controlled Trial (Control of Hypertension in Pregnancy Study): Is Severe Hypertension Just an Elevated Blood Pressure? Hypertension. 2016; 68:1153-1159.

11. Panaitescu AM, Syngelaki A, Prodan N, Akolekar R, Nicolaides KH. Chronic hypertension and adverse pregnancy outcomes: a cohort study. Ultrasound Obstet Gynecol. 2017 Apr 23. https://doi.org/10.1002/uog.17493. [Epub ahead of print].

12. Ohkuchi A, Iwasaki R, Suzuki H, Hirashima C, Takahashi K, Usui R, Matsubara S, Minakami H, Suzuki M. Normal and high-normal blood pressures, but not body mass index, are risk factors for the subsequent occurrence of both preeclampsia and gestational hypertension: a retrospective cohort study. Hypertens Res. 2006; 29:161-7.

13. Mabuchi A, Yamamoto R, Ishii K, Kakubari R, Takaoka S, Muto H, Mitsuda N. Significance of high-normal blood pressure during early second trimester for predicting the onset of hypertensive disorders in pregnancy. Hypertens Pregnancy. 2016; 35:234-41.

14. Vonck S, Oben J, Staelens AS, Lanssens D, Tomsin K, Gyselaers W. Normal and abnormal blood pressures in early pregnancy: are we using the right cut off values? J Matern Fetal Neonatal Med. 2016; 29:9.

15. Lisonkova $\mathrm{S}$, Joseph KS. Incidence of preeclampsia: risk factors and outcomes associated with early- versus lateonset disease. Am J Obstet Gynecol. 2013; 209:544. e1-12.

16. Catov JM, Ness RB, Kip KE, Olsen J. Risk of early or severe pre-eclampsia related to preexisting conditions. Int J Epidemiol. 2007; 36:412-9.

17. McCowan LM, Buist RG, North RA, Gamble G. Perinatal morbidity in chronic hypertension. Br J Obstet Gynaecol. 1996; 103:123-9.

18. Bustamante Helfrich B, Chilukuri N, He H, Cerda SR, Hong X, Wang G, Pearson C, Burd I, Wang X. Maternal vascular malperfusion of the placental bed associated with hypertensive disorders in the Boston Birth Cohort. Placenta. 2017; 52:106-113.

19. Melchiorre K, Sutherland GR, Liberati M, Thilaganathan B. Maternal cardiovascular impairment in pregnancies complicated by severe fetal growth restriction. Hypertension. 2012; 60:437-443.

20. Bramham K, Parnell B, Nelson-Piercy C, Seed PT, Poston L, Chappell LC. Chronic hypertension and pregnancy outcomes: systematic review and meta-analysis. BMJ. 2014; 348:g2301.
21. Caruso A, Ferrazzani S, De Carolis S, Lucchese A, Lanzone A, Paradisi G. Carbohydrate metabolism in gestational diabetes: effect of chronic hypertension. Obstet Gynecol. 1999; 94:556-561.

22. Hu FB, Stampfer MJ. Insulin resistance and hypertension: the chicken-egg question revisited. Circulation. 2005; 112:1678-1680.

23. Naderi S, Tsai SA, Khandelwal A. Hypertensive Disorders of Pregnancy. Curr Atheroscler Rep. 2017; 19:15.

24. People website. China's average childbearing age from 26.29 years postponed to 28.18 years old. 2014. Available at: http://js.people.com.cn/html/2014/03/24/297207.html (Accessed March 24, 2014).

25. Li C, Liu Y, Zhang W. Joint and Independent Associations of Gestational Weight Gain and Pre-Pregnancy Body Mass Index with Outcomes of Pregnancy in Chinese Women: A Retrospective Cohort Study. PLoS One. 2015; 10:e0136850.

26. Ohkuchi A, Iwasaki R, Suzuki H, Hirashima C, Takahashi K, Usui R, Matsubara S, Minakami H, Suzuki M. Normal and high-normal blood pressures, but not body mass index, are risk factors for the subsequent occurrence of both preeclampsia and gestational hypertension: a retrospective cohort study. Hypertens Res. 2006; 29:161-7.

27. Cunningham FG, Leveno KJ, Bloom SL, Hauth JC, Rouse DJ. Pregnancy hypertension. Williams Obstetrics, 23rd edition. McGraw-Hill p.706-756.

28. Sacks DA, Hadden DR, Maresh M, Deerochanawong C, Dyer AR, Metzger BE, Lowe LP, Coustan DR, Hod M, Oats JJ, Persson B, Trimble ER; HAPO Study Cooperative Research Group. Frequency of gestational diabetes mellitus at collaborating centers based on IADPSG consensus panelrecommendedcriteria: the Hyperglycemia and Adverse Pregnancy Outcome (HAPO) Study. Diabetes Care. 2012; 35:526-528.

29. WHO. Physical status: the use and interpretation of anthropometry. Geneva: World Health Organization, 1995.

30. Institute of Medicine \& National Research Council Committee to Reexamine, I. O. M. P. W. G. (2009) In Weight Gain During Pregnancy: Reexamining the Guideline (eds Rasmussen KM, Yaktine AL). National Academies Press (US) National Academy of Sciences. 